\title{
Endoscopic ultrasound-guided hepaticogastrostomy and antegrade clearance of biliary lithiasis in patients with surgically-altered anatomy
}

\section{(๑) $\odot \ominus$}

\author{
Authors \\ Amy Hosmer ${ }^{1}$, Mohamed M Abdelfatah ${ }^{2}$, Ryan Law ${ }^{1}$, Todd H. Baron ${ }^{3}$
}

Institutions

1 Division of Gastroenterology \& Hepatology, University of Michigan, Ann Arbor, Michigan, United States

2 Division of Gastroenterology and Hepatology, East Carolina University, Greenville, North Carolina, United States

3 Division of Gastroenterology and Hepatology, University of North Carolina, Chapel Hill, North Carolina, United States

submitted 3.11 .2017

accepted after revision 7.11.2017

\author{
Bibliography \\ DOI https://doi.org/10.1055/s-0043-123188 | \\ Endoscopy International Open 2018; 06: E127-E130 \\ (c) Georg Thieme Verlag KG Stuttgart · New York \\ ISSN 2364-3722
}

Corresponding author

Todd H. Baron, MD, 130 Mason Farm Road, CB 7080, Chapel Hill, NC 27599

Fax: 984-974-0744

todd_baron@med.unc.edu

\section{ABSTRACT}

Background and study aims Endoscopic retrograde cholangiography (ERC) in patients with complex surgically-altered anatomy (SAA) is technically demanding and has limitations. Developments in EUS-guided procedures allow alternative approaches for patients with altered gastrointestinal anatomy and biliary lithiasis.

Patients and methods Single-center, retrospective review of prospectively entered patients with SAA who underwent EUS-guided hepaticogastrostomy (HGS) followed by an interval antegrade endoscopic clearance of biliary lithiasis.

Results 9 patients with Roux-en-Y anatomy underwent HGS to allow clearance of biliary lithiasis after a mean of 2.5 procedures. Technical success was achieved in $100 \%$ of patients utilizing subsequent antegrade endoscopic techniques after HGS including: balloon sweep (9), transpapillary balloon dilation (8), cholangioscopy with electrohydraulic lithotripsy (4), and mechanical lithotripsy (1). HGS stents were removed in all patients. 1 adverse event (cholangitis) occurred after cholangioscopy and prolonged intraductal electrohydraulic lithotripsy.

Conclusion EUS-guided antegrade therapy for the management of biliary lithiasis in patients with altered gastrointestinal anatomy appears efficacious with a low risk of adverse events. These preliminary results suggest this approach should be considered at centers with available expertise.

\section{Introduction}

The role of therapeutic endoscopic ultrasound (EUS) in management of gastrointestinal disorders continues to evolve. While originally imagined as a diagnostic tool, the therapeutic capability of EUS has expanded significantly over recent years, most notably in techniques for EUS-guided biliary interventions. EUS-guided biliary interventions can be pursued in patients with an inaccessible papilla due to surgically-altered gastrointestinal anatomy (SAA), or in patients with biliary obstruction and incomplete biliary drainage utilizing conventional endoscopic methods (i.e. ERCP) [1].
Endoscopic retrograde cholangiography (ERC) in patients with SAA is technically difficult, particularly following Rouxen-Y (RY) surgery such as gastric bypass (RYGB) and hepaticojejunostomy (RYHJ). Balloon enteroscopy-assisted ERC (BAERC) $[2,3]$ can be used for SAA, though the long length and limited working channels of these enteroscopes prevent the use of many standard accessories, including lithotripsy devices and the ability to use cholangioscopy-assisted lithotripsy (laser and electrohydraulic lithotripsy, EHL). In addition, even when the pancreaticobiliary limb can be successfully traversed to reach the second portion of the duodenum in RYGB patients, cannulation of native papillae is challenging due to the for- 


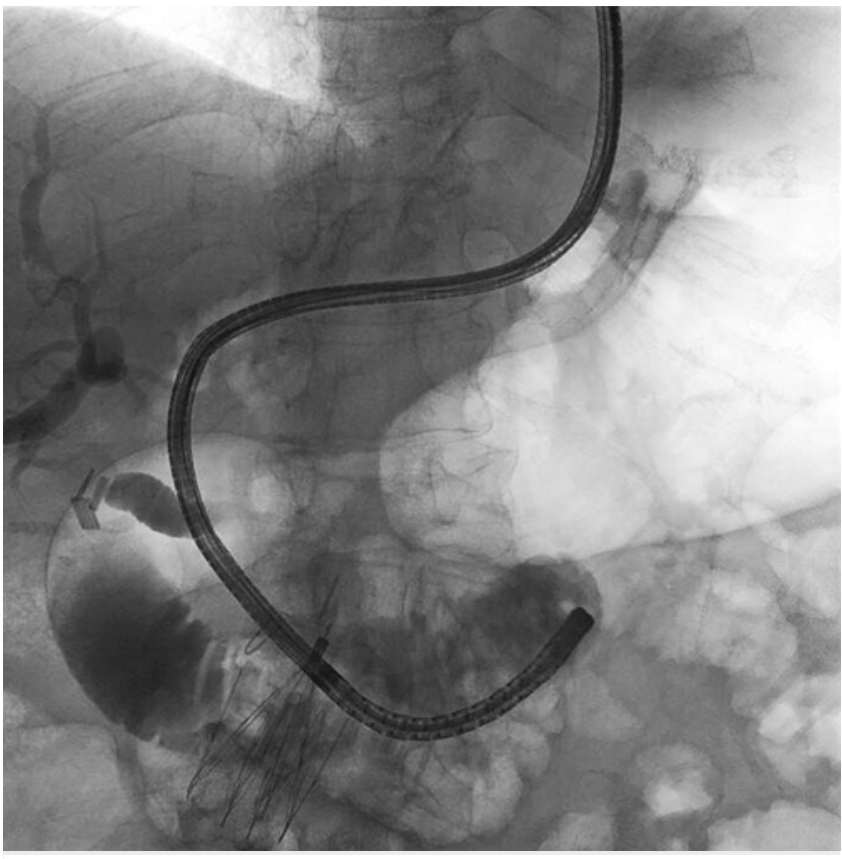

- Fig. 1 Complete cholangioscopy performed in an antegrade fashion to evaluate and treat biliary lithiasis.

ward optics and working channel of the enteroscope, and lack of an elevator. As such, patients often undergo percutaneous transhepatic biliary drainage (PTBD) to provide decompression in this setting. After placement, the PTBD catheter remains in place for a minimum of 4 weeks and often much longer. Percutaneous drainage catheters are painful, require patient care and may inadvertently dislodge. Thus, further development of EUS-guided techniques for internal drainage have the potential advantage of improved quality of life [4].

Recent literature suggests that therapeutic EUS can be considered for the management of patients with SAA and biliary lithiasis [5]. Herein we report our experience using EUS-guided hepaticogastrostomy (HGS) to facilitate subsequent antegrade ductal clearance in patients with Roux-en-Y anatomy and biliary lithiasis.

\section{Patients and methods}

A prospectively maintained EUS database was reviewed to identify consecutive patients with SAA who underwent EUS-guided HGS for the management of biliary lithiasis from February 2016 to May 2017 at University of North Carolina-Chapel Hill. Written informed consent and IRB approval was obtained on all patients. Patient demographics, procedural information and clinical details were abstracted from the electronic medical record and endoscopy database.

EUS-guided antegrade therapy was performed by 1 endoscopist (T. H. B.) using a standard oblique-viewing linear echoendoscope (Olympus, Center Valley, PA) with patients under general anesthesia in the supine position. Endoscopic HGS was created by transgastric/transjejunal puncture using fine needle injection, confirmation of position within the bile duct, guidewire

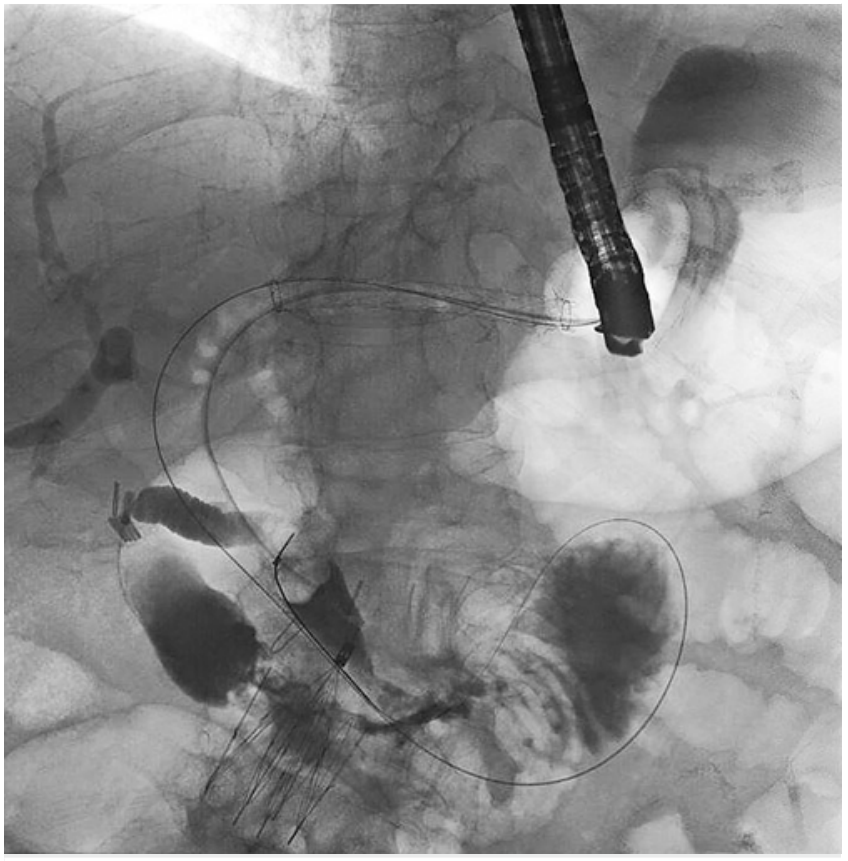

- Fig. 2 Antegrade sweeping of the common bile duct utilizing an extraction balloon.

placement, balloon dilation $(4-6 \mathrm{~mm})$, and placement of a fully-covered self-expandable metal biliary stent (SEMS) of 8or $10-\mathrm{mm}$ diameters and lengths of $6 \mathrm{~cm}$ to $10 \mathrm{~cm}$ (Viabil, W.L. Gore, Flagstaff, AZ). In all patients a 7Fr plastic stent was placed through the SEMS. In a second procedure, stone clearance was performed with a standard duodenoscope positioned in the stomach or jejunum under fluoroscopy through the HGS using antegrade biliary techniques and accessories after antegrade guidewire passage into the duodenum or jejunum, and balloon dilation of the native papilla or HJ. Stone clearance was facilitated by antegrade cholangioscopy using digital cholangioscopy (SpyGlass ${ }^{\mathrm{TM}}$ DS, Boston Scientific, Marlborough, MA) passed through a duodenoscope or via direct peroral antegrade cholangioscopy using small-caliber [5.4mm] forward-viewing upper endoscopes (Olympus GIF-XP series) ( $\triangleright$ Fig. 1). Intraductal electrohydraulic+/- mechanical lithotripsy, and antegrade sweeping using a standard extraction balloon were performed as needed ( $\mathbf{F i g} \mathbf{2} \mathbf{2}$ ).

Technical success was defined as creation of an EUS-guided HGS followed by subsequent complete antegrade clearance of bile duct stones. The definition and severity of adverse events were based on previously published guidelines [6].

\section{Results}

9 patients (mean age 60 years; $89 \%$ women) with prior Rouxen-Y surgery underwent hepaticogastrostomy (HGS) to facilitate management of biliary lithiasis ( $\triangleright$ Table 1$)$. The post-surgical anatomy in our cohort was: RYGB (6), RYHJ (2), and Rouxen-Y antrectomy (1). 5 patients underwent EUS-guided HGS during inpatient stays, as they had been hospitalized for acute biliary disease prior to undertaking therapy. 2 patients under- 
- Table 1 Patients undergoing successful EUS-guided antegrade therapy.

\begin{tabular}{|c|c|c|c|c|c|c|}
\hline $\begin{array}{l}\text { Patient } \\
\text { number }\end{array}$ & Anatomy & $\begin{array}{l}\text { Time from EUS to } \\
\text { antegrade stone } \\
\text { therapy (days) }\end{array}$ & $\begin{array}{l}\text { Cholan- } \\
\text { gioscopy }\end{array}$ & $\begin{array}{l}\text { Downstream } \\
\text { Balloon Dila- } \\
\text { tion }(\mathrm{mm})\end{array}$ & Additional methods & $\begin{array}{l}\text { Time from stone } \\
\text { clearance to stents } \\
\text { removal (days) }\end{array}$ \\
\hline 1 & RYGB & 98 & $\mathrm{Y}$ & 18 & EHL, balloon extraction & $\mathrm{N} / \mathrm{A}$ \\
\hline 2 & RY antrectomy & 9 & Y & 10 & EHL, balloon extraction & 0 \\
\hline 3 & $\mathrm{HJ}$ & 34 & $\mathrm{Y}$ & 10 & EHL, balloon extraction & 0 \\
\hline 4 & RYGB & 35 & $\mathrm{Y}$ & 12 & Balloon extraction & 0 \\
\hline 5 & RYGB & 30 & Y & 12 & Balloon extraction & 0 \\
\hline 6 & RYGB & 38 & Y & $N / A$ & Balloon extraction & 0 \\
\hline 7 & RYGB & 42 & Y & 10 & Balloon extraction & 0 \\
\hline 8 & RYGB & 41 & $\mathrm{Y}$ & 15 & EHL, ML, balloon extraction & 0 \\
\hline 9 & HJ & 20 & $\mathrm{~N}$ & 10 & Balloon extraction & 0 \\
\hline
\end{tabular}

went failed BAERC prior to undergoing EUS-guided HGS. Technical success was achieved in $100 \%$ of patients. Complete ductal clearance was accomplished using various techniques: antegrade balloon sweeps (9), transpapillary balloon dilation (8), cholangioscopy with electrohydraulic lithotripsy (4), and mechanical lithotripsy (1). 4 additional patients underwent cholangioscopy to confirm duct clearance. In 8 patients, HGS stents were removed immediately following duct clearance ( 1 patient with fully-covered SEMS in situ for ongoing treatment of distal bile duct stricture) using grasping devices or standard polypectomy snares. The mean time between HGS placement and removal of all stents was 31 days (range 9-42 days). A mean of 2.5 procedures (range $2-4$ procedures) were required per patient, including initial HGS and subsequent procedures to complete stone clearance. 1 mild severe adverse event (cholangitis requiring 3 days of hospitalization) occurred after cholangioscopy using a small caliber forward-viewing endoscope. In this patient prolonged intraductal electrohydraulic lithotripsy was performed. We believe the large volume of saline and inability of fluid to escape from the biliary tree around the stent $(5.4 \mathrm{~mm}$ endoscope within an $8 \mathrm{~mm}$ stent) resulted in high intraductal filling pressures and subsequent bacterial translocation into the liver.

6 patients with native papillae were given rectal indomethacin for prevention of post-ERC pancreatitis. No post-ERC pancreatitis occurred despite balloon dilation of a native papilla up to $18 \mathrm{~mm}$ ( $>$ Table $\mathbf{1}$ ). Attempts at HGS failed in 2 patients with RYGB anatomy due to non-dilated intrahepatic ducts. Following HGS failure, both patients underwent BAERC (technical success in 1 patient).

Several patients complained of bile reflux symptoms while the transgastric stents were in place. These symptoms resolved following stent removal.

\section{Discussion}

When utilizing EUS-guided antegrade therapy for the management of biliary lithiasis, our data demonstrate a high rate of technical success (100\%) with an acceptable risk of adverse events (11\%). This two-phase technique utilizes EUS-guided HGS to provide initial decompression of biliary obstruction, followed by a subsequent intervention using ERC techniques to achieve ductal clearance. We performed large diameter ( $\geq 10 \mathrm{~mm}$, up to $18 \mathrm{~mm}$ ) balloon dilation of native papillae in 8 of 9 patients without the development of post-ERC pancreatitis. In selected patients, antegrade cholangioscopy facilitated stone extraction and/or confirmation of stone clearance. A similar two-stage procedure has been previously published for the management of benign, post-surgical biliary strictures using HGS followed by antegrade balloon dilation $+/$ - stent placement [7].

EUS-guided pancreaticobiliary therapy is performed in selected tertiary care centers worldwide $[8,9]$. These procedures require expertise in EUS and ERCP, and can serve as an alternative to percutaneous therapy [10] or ERC using long-length endoscopes. In 1 retrospective study from outside the U.S. [5], EUS-guided antegrade therapy for biliary lithiasis was performed in patients with SAA, though none of these patients had RYGB anatomy. The results of this study were similar with a technical success rate of $72 \%$ and adverse event rate of $17 \%$.

Although there are no dedicated SEMS for creation of hepaticogastric anastomoses in the U.S., we found the current stent to be well suited to this procedure. We avoided electrocautery during stent placement and the long stent length anchored the position such that intraperitoneal migration did not occur.

We performed antegrade cholangioscopy using either a digital cholangioscope passed through a duodenoscope or via direct peroral antegrade cholangioscopy with small caliber upper endoscopes. We noted that the latter approach was easier 
when the HGS SEMS was removed, as it limited the angle of entry into the left intrahepatic system.

The senior author's current approach to patients with SAA and biliary lithiasis is to proceed directly to EUS-guided antegrade therapy in patients with RYGB anatomy when dilated intrahepatic ducts are seen at EUS, especially when urgent biliary drainage is needed. A recently proposed alternative method for management of biliary lithiasis in patients with Roux-en-Y anatomy involves creation of EUS-guided gastrogastrostomy or jejunogastrostomy, with subsequent delayed routine ERC via the fistulous connection. While available data suggest a high-rate of technical success and low risk of adverse events, this technique has limitations [11]. The most widely accepted iteration of this technique also involves 2 steps, as stent dislodgement into the peritoneum using adult duodenoscopes has occurred when same-day ERC is attempted after creation of the EUSguided gastrogastric or jejunogastric anastomoses [12]. Additionally, following stent removal these translumenal anastomoses may remain patent following management of bile duct stones, and lead to weight regain due to failed spontaneous closure of the gastrogastrostomy.

The advantages of endoscopic over percutaneous treatment include not only the avoidance of painful external drains that limit quality of life, but the potential for these endoscopic procedures (initial and subsequent) to be performed in an outpatient setting.

\section{Conclusion}

In conclusion, we believe EUS-guided antegrade hepaticogastrostomy and antegrade endoscopic therapy for treatment of biliary lithiasis in patients with surgically-altered anatomy is a safe and effective alternative to percutaneous transhepatic therapy when performed in centers with available expertise.

\section{Competing interests}

Todd H. Baron is a speaker and consultant for Medtronic, $\mathrm{BSCl}$, Cook and Olympus.
References

[1] Wang K, Zhu J, Xing L et al. Assessment of efficacy and safety of EUSguided biliary drainage: a systematic review. Gastrointest Endosc 2016; 83: $1218-1227$

[2] Cai JX, Diehl DL, Kiesslich R et al. A multicenter experience of throughthe-scope balloon-assisted enteroscopy in surgically altered gastrointestinal anatomy. Surg Endosc 2017; 31: $2753-2762$

[3] Inamdar S, Slattery E, Sejpal DV et al. Systematic review and meta-analysis of single-balloon enteroscopy-assisted ERCP in patients with surgically altered GI anatomy. Gastrointest Endosc 2015; 82: 9-19

[4] Law R, Perez-Miranda M. Is it time to stop using percutaneous transhepatic biliary drainage? Endoscopy 2017; 49: 521 - 523

[5] Iwashita T, Nakai Y, Hara K et al. Endoscopic ultrasound-guided antegrade treatment of bile duct stone in patients with surgically altered anatomy: a multicenter retrospective cohort study. J Hepatobiliary Pancreat Sci 2016; 23: 227-233

[6] Cotton PB, Eisen GM, Aabakken L et al. A lexicon for endoscopic adverse events: report of an ASGE workshop. Gastrointest Endosc 2010; 71: $446-454$

[7] Miranda-Garcia P, Gonzalez JM, Tellechea JI et al. EUS hepaticogastrostomy for bilioenteric anastomotic strictures: a permanent access for repeated ambulatory dilations? Results from a pilot study Endosc Int Open 2016; 4: E461 - 465

[8] Committee AT, Maple JT, Pannala R et al. Interventional EUS (with videos). Gastrointest Endosc 2017; 85: 465-481

[9] Jirapinyo P, Lee LS. Endoscopic Ultrasound-Guided Pancreatobiliary Endoscopy in Surgically Altered Anatomy. Clin Endosc 2016; 49: $515-$ 529

[10] Sharaiha RZ, Khan MA, Kamal F et al. Efficacy and safety of EUS-guided biliary drainage in comparison with percutaneous biliary drainage when ERCP fails: a systematic review and meta-analysis. Gastrointest Endosc 2017; 85: 904-914

[11] Ngamruengphong S, Nieto J, Kunda R et al. Endoscopic ultrasoundguided creation of a transgastric fistula for the management of hepatobiliary disease in patients with Roux-en-Y gastric bypass. Endoscopy 2017; 49: 549-552

[12] Tyberg A, Nieto J, Salgado S et al. Endoscopic Ultrasound (EUS)-Directed Transgastric Endoscopic Retrograde Cholangiopancreatography or EUS: Mid-Term Analysis of an Emerging Procedure. Clin Endosc 2017; 50: 185-190 